\title{
Piezoelectricity in Polarized Poly(vinylidene fluoride) Films
}

\author{
Eiichi Fukada and Tsutau SAKURAI* \\ The Institute of Physical and Chemical Research, Wako, Saitama, Japan.
}

(Received March 31, 1971)

\begin{abstract}
Piezoelectric films of poly(vinylidene fluoride) were prepared by applying a static electric field of about $500 \mathrm{kV} / \mathrm{cm}$ to seven-times elongated films at $80^{\circ} \mathrm{C}$ for a few hours. The tensile stress $T$ produced the polarization $P$ normal to the surface of the film. The real and imaginary components of the complex piezoelectric strainconstant $d=d^{\prime}-i d^{\prime \prime}=P / T$ were determined at temperatures from -140 to $+160^{\circ} \mathrm{C}$. The relation between the piezoelectric constant $d^{\prime}$ and the angle $\theta$ between the direction of stress and the orientation axis is given by $d^{\prime}=d_{31}^{\prime} \cos ^{2} \theta+d_{32}^{\prime} \sin ^{2} \theta$, where the 1 -axis and 2-axis are parallel and perpendicular to the direction of orientation and the 3-axis is normal to the film plane. The values of $d_{31}^{\prime}$ and $d_{52}^{\prime}$ at room temperature were approximately $6 \times 10^{-8}$ and $1.5 \times 10^{-8} \mathrm{cgs}$ esu, respectively. The piezoelectric dispersions occurred at four temperatures at which the elastic dispersions also took place.

KEY WORDS Poly(vinylidene fluoride)/ Piezoelectricity / Electret / Orientation / Temperature Dispersion / Anisotropy /
\end{abstract}

The piezoelectric effect has been reported in a number of semicrystalline polymers, including biological polymers such as cellulose ${ }^{1}$ and collagen ${ }^{2}$ and synthetic polymers such as poly $\left(\gamma\right.$-methyl-L-glutamate) ${ }^{3}$ and isotactic poly (propylene oxide $)^{4}$. A shear stress applied to the polymer generates an electrical polarization in the direction perpendicular to the plane of the stress. The origin of such piezoelectricity is ascribed to the piezoelectric properties of the crystalline regions of the polymer.

A new type of piezoelectricity in polymers has been discovered recently by Kawai. ${ }^{5,6}$ The polymer films are highly elongated and are subjected to a strong static electric field at a high temperature. After such a poling process, many kinds of films are semipermanently polarized. Such electret films are usually piezoelectric. The anisotropy of the new piezoelectricity is quite different to that described in a previous paper. ${ }^{9}$ Poly (vinylidene fluoride) $\left(\mathrm{PVF}_{2}\right)$ produced the highest piezoelectric effect among polymers investigated. ${ }^{6}$

Nakamura and Wada $^{7}$ have reported the piezoelectricity in roll-drawn films of $\mathrm{PVF}_{2}$ and related the effect to the electrostriction and the antisymmetrical distribution of space charges.

* Department of Physics, Gakushuin University, Mejiro, Tokyo.
Edelman, et al., ${ }^{8}$ have also found a large piezoelectric effect in poly (vinyl chloride).

The present paper describes the investigations on the piezoelectric properties of elongated and polarized $\mathrm{PVF}_{2}$, particularly, the anisotropy and temperature dependence of the complex piezoelectric constant.

\section{EXPERIMENTAL}

Films of poly(vinylidene fluoride) were furnished by Kureha Chemical Industry Co., Ltd. The film had been uniaxially elongated to seven times its original length and the thickness was $0.05 \mathrm{~mm}$. For the purpase of determining the anisotropy of the piezoelectric effect, circular samples of about $10 \mathrm{~mm}$ diameter were prepared and very thin brass foils were cemented to the center of the film as electrodes with the alcoholic solution of schellac. For the measurement of temperature dependence, the rectangular samples, $6 \times 10 \mathrm{~mm}$, were cut along either parallel or perpendicular to the orientation axis of the film. Thin brass foils, $4 \times 4 \mathrm{~mm}$, were joined to the central part of the sample and silver ribbons as lead wires were joined to the electrodes.

The samples were heated to about $80^{\circ} \mathrm{C}$ in a thermostat and were given a high D.C. voltage 
up to $5 \mathrm{kV}$ for two or three hours, and then gradually cooled down to room temperature for several hours under a high electric field. The films then attained the electret state ${ }^{10,11}$ at room temperature. The negative charge was observed on the surface of the film which contacted with the negative electrode during the poling procedure. The positive charge was observed on the opposite surface. This means that homocharges were developed on the surfaces of the electrified films.

Figure 1 shows the rectangular coordinates assigned to the film. The $z$-axis is perpendicular to the surface of the film and its sense is

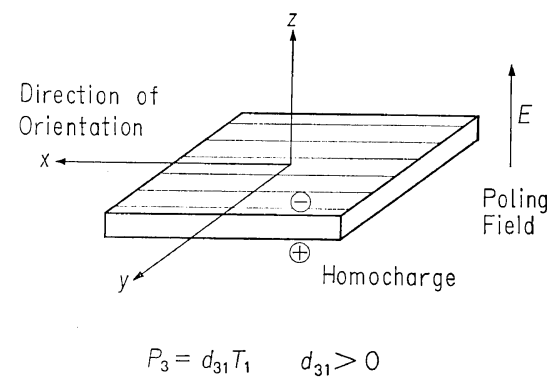

Figare 1. Coordinates of the specimen and the direction of the poling field. Homocharges are developed on the specimen.

parallel with the direction of the poling electric field. The $x$-axis is assigned to the direction of elongation of the film. The $y$-axis is perpendicular to both the $x$ - and $z$-axes.

We have observed that the tension $T_{1}$ in the positive $x$ direction produces the positive polarization $P_{3}$ in the $z$ direction. The piezoelectric strain-constant, $d_{31}$, which is defined by

$$
P_{3}=d_{31} T_{1}
$$

is, therefore, positive. The piezoelectric strainconstant, $d_{32}$, which relates $P_{3}$ to the tension $T_{2}$ in the $y$ direction, is also positive.

The preparation of piezoelectric electret films needs some caution. Electrical break-down during the poling process must be carefully avoided. Generally the higher electric field produces the higher polarization and thus the higher piezoelectric effect. The magnitudes of $d_{31}$ and $d_{32}$ at room temperature were examined by varying the poling field and the results
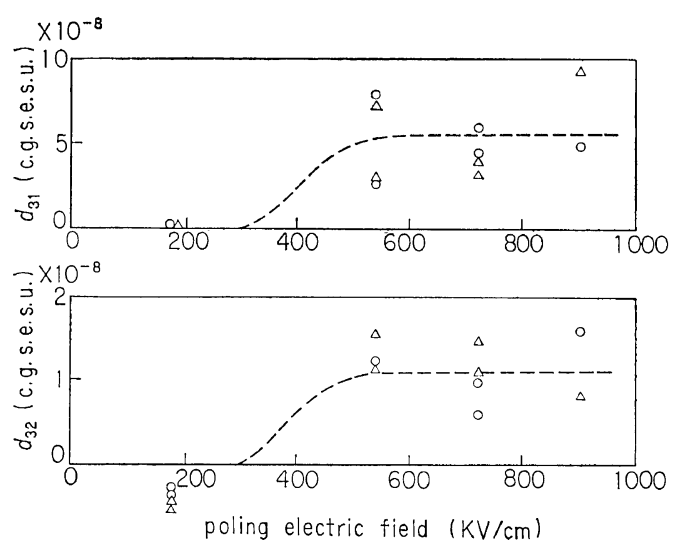

Figure 2. Dependence of piezoelectric strain-constants $d_{31}$ and $d_{32}$ on the poling field.

are shown in Figure 2. It was found that the threshold value exists for the electric field at about $300 \mathrm{kV} / \mathrm{cm}$. Very little piezoelectric effect was observed for the films treated under the electric field lower than about $300 \mathrm{kV} / \mathrm{cm}$. Existence of the threshold electric field has also been reported for the relation between the surface polarization and the poling electric field. ${ }^{12}$ In the present study, an electric field of about $500 \mathrm{kV} / \mathrm{cm}$ was employed as the poling field.

The poling temperature is also important. Kawai $^{5}$ reported that $80^{\circ} \mathrm{C}$ is the most appropriate temperature for forming the electret. It is known that crystalline dispersion takes place at about $80^{\circ} \mathrm{C}$ for $\mathrm{PVF}_{2}{ }^{13,14}$. Very high temperature brings about a large increase of electrical conductivity and thus the electric break-down readily takes place. We have chosen $80^{\circ} \mathrm{C}$ as the poling temperature in the present study.

Complex piezoelectric constants and their temperature variations were determined by an apparatus described in detail in a previous paper. $^{3}$ The film was clamped at both edges and was given a sinusoidal oscillation at $20 \mathrm{~Hz}$. The electrical voltage produced between the electrodes of the sample was compensated to nil by applying an electric voltage derived from the coil current in the driver, which was proportional to the stress in the film. The electrical polarization in the film was obtained from this compensating voltage. From 


\section{E. Fukada and T. Sakurai}

the ratio of the polarization $P$ to the stress $T$ and the phase angle between the polarization and the stress, the real and imaginary components of complex piezoelectric strain-constant $d=d^{\prime}-i d^{\prime \prime}$ were determined.

\section{ANISOTROPY IN PIEZOELECTRICITY}

The dependence of the piezoelectric strain- constant on the direction of the applied stress with regard to the orientation axis of the film was studied in the circular samples. Figures 3 and 4 show the results for $d^{\prime}$ and $d^{\prime \prime}$. The measurements were carried out 1 and 105 day after the poling process of the sample. Some decay of piezoelectric effect is observed for this sample. Kawai has reported, however, a good stability for the piezoelectric constant in

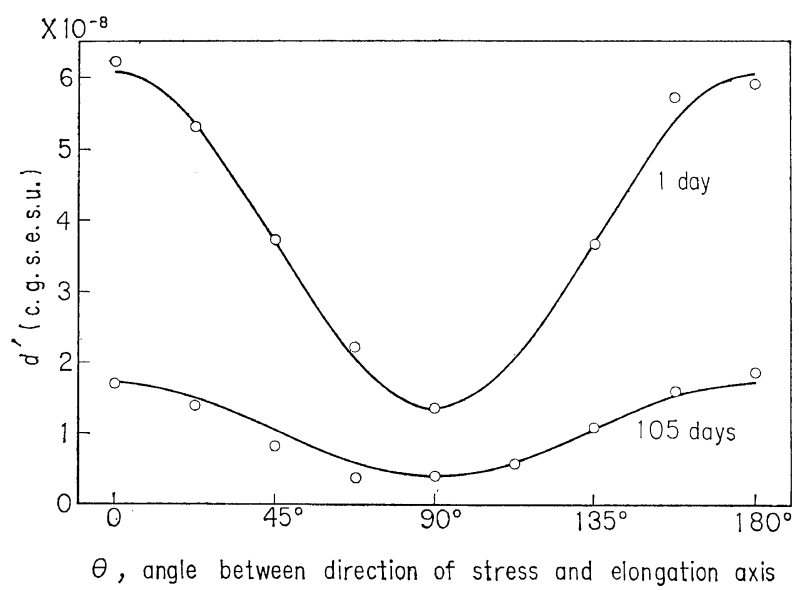

Figure 3. Dependence of the real piezoelectric strain-costant $d^{\prime}$ on the angle between the direction of stress and the axis of elongation. Measurements were carried out 1 and 105 day after the poling procedure.

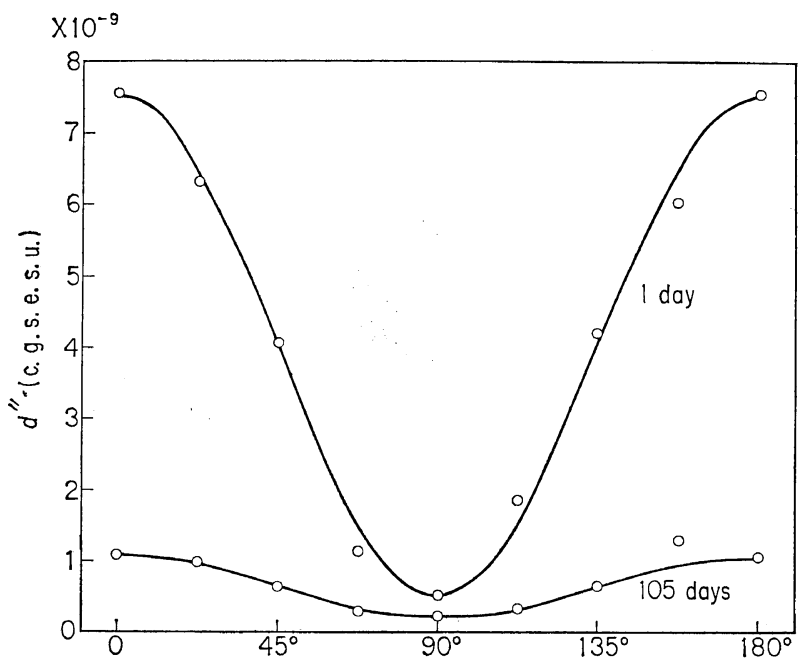

$\theta$, angle between direction of stress and elongation axis

Figure 4. Dependence of the imaginary piezoelectric strain-constant $d^{\prime \prime}$ on the angle between the direction of stress and the axis of elongation. Measurements were carried out 1 and 105 day after the poling procedure. 
well treated films of $\mathrm{PVF}_{2}{ }^{5}$ The constants at $\theta=0^{\circ}$ and $90^{\circ}$ are $d_{31}$ and $d_{32}$, respectively. The solid lines in Figure 3 and 4 are drawn from the following equations,

$$
\begin{gathered}
d^{\prime}=d_{31}^{\prime} \cos ^{2} \theta+d_{32}^{\prime} \sin ^{2} \theta \\
d^{\prime \prime}=d_{32}^{\prime \prime} \cos ^{2} \theta+d_{32}^{\prime \prime} \sin ^{2} \theta
\end{gathered}
$$

The phase angles between the polarization and the stress are given by $\tan \delta_{31}=d_{31}^{\prime \prime} / d_{31}^{\prime}=0.13$ at 1 day and 0.06 at 105 day and $\tan \delta_{32}=d_{32}^{\prime \prime} / d_{32}^{\prime \prime}=$ 0.04 at 1 day and 0.05 at 105 day.

\section{TEMPERATURE DISPERSION}

Figure 5 illustrates the temperature variation of $d_{31}^{\prime}$ and $d_{31}^{\prime \prime}$ for a $\mathrm{PVF}_{2}$ film, seven times elongated and polarized under $600 \mathrm{kV} / \mathrm{cm}$ at $80^{\circ} \mathrm{C}$. With the rise of temperature both constants increase with peaks or shoulders at four temperatures. The peaks of $d_{31}^{\prime \prime}$ are observed at about $-100,-30,70$, and $140^{\circ} \mathrm{C}$.

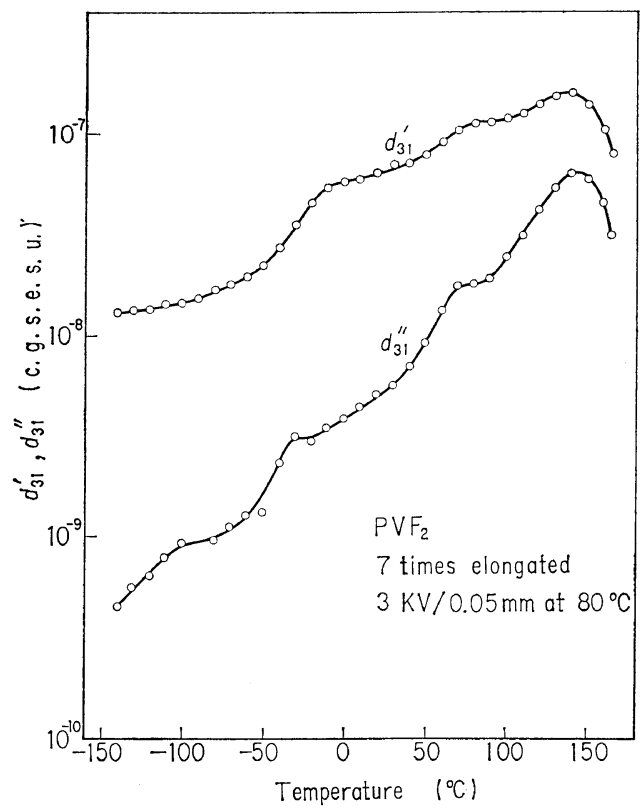

Figure 5. Temperature dependence of the piezoelectric strain-constants, $d_{31}^{\prime}$ and $d_{31}^{\prime \prime}$.

Figure 6 illustrates the variation of $d_{32}^{\prime}$ and $d_{32}^{\prime \prime}$ for the same sample of $\mathrm{PVF}_{2}$ with temperature above $-70^{\circ} \mathrm{C}$. The peaks of $d_{32}^{\prime \prime}$ are observed at about $-30,70$ and $130^{\circ} \mathrm{C}$.

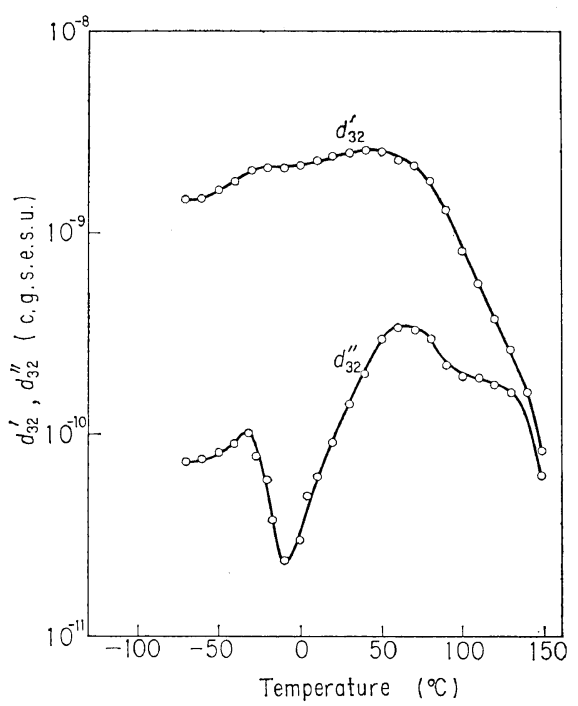

Figure 6. Temperature dependence of the piezoelectric strain-constants, $d_{32}^{\prime}$ and $d_{32}^{\prime \prime}$.

For the purpose of comparison between piezoelectric and elastic properties, the temperature dependence of dynamic elastic modulus $E_{1}{ }^{\prime}$ and loss modulus $E_{1}^{\prime \prime}$ was determined at $10 \mathrm{~Hz}$ by a viscoelastorecorder (VER), ${ }^{15}$ and the results are shown in Figure 7. The direction of the

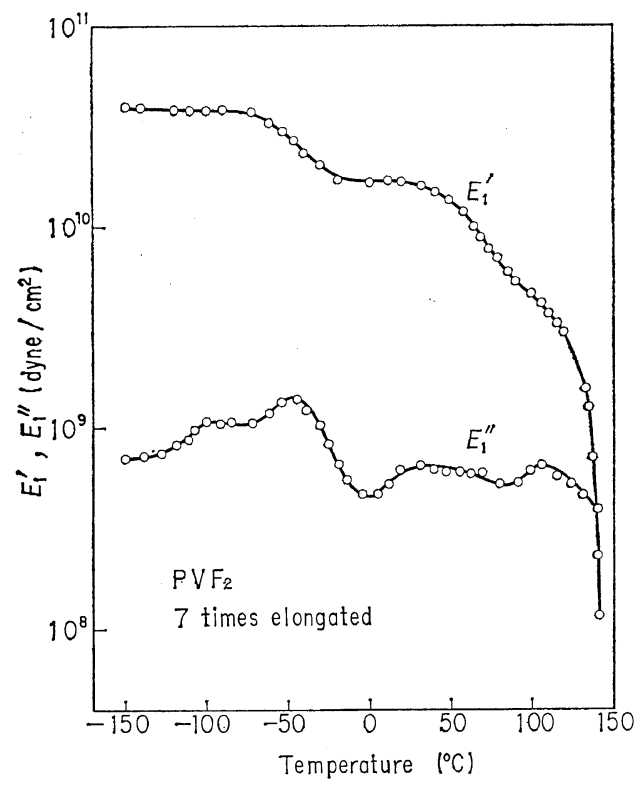

Figure 7. Temperature dependence of the elastic modulus $E_{1}{ }^{\prime}$ and the loss modulus $E_{1}{ }^{\prime \prime}$. 


\section{E. FukAda and T. SAKurai}

applied sinusoidal stress was in the orientation axis of the film. The peaks of $E_{1}^{\prime \prime}$ are seen at four temperatures of about $-100,-40,50$, and $110^{\circ} \mathrm{C}$. It is obvious that the viscoelastic dispersions take place at the same temperature as those of the piezoelectric dispersions. According to previous studies, ${ }^{13,14}$ the nature of the dispersions from the low to high temperatures has been ascribed to the local relaxation $\left(-100^{\circ} \mathrm{C}\right)$, the primary dispersion $\left(-40^{\circ} \mathrm{C}\right)$, the crystalline dispersions in the $\alpha$-form $\left(70^{\circ} \mathrm{C}\right)$ and $\beta$-form $\left(110^{\circ} \mathrm{C}\right)$ crystallites, respectively.

\section{DISCUSSION}

The piezoelectric effect in the polarized $\mathrm{PVF}_{2}$ films is different in many respects to those observed in the previously studied piezoelectric polymers such as synthetic polypeptides. One of the largest differences is the anisotropy of the effect. In the oriented films of polypetides, for example, only the shear stress produces electrical polarization. Denoting $\theta$ the angle between the direction of the extensional stress and the elongation axis of the film, the piezoelectric constant $d$ is expressed by

$$
d=\left(d_{36} / 2\right) \sin 2 \theta,
$$

where the coordinates shown in Figure 1 are also used for the film. The extensional stress at $\theta=45^{\circ}$ or $135^{\circ}$ produces the maximum polarization. On the other hand, the piezoelectric anisotropy in the polarized $\mathrm{PVF}_{2}$ films is expressed by eq 2 . The extensional stress at $\theta=$ $0^{\circ}$ produces the maximum polarization.

The piezoelectric stress-constant $e$ is defined as the polarization, $P$, produced by strain, $S$, under zero electric field, i.e.,

$$
P=e S, \quad(E=0)
$$

or the stress, $T$, produced by electric field, $E$, under zero strain, i.e.,

$$
T=e E, \quad(S=0)
$$

If a sinusoidal electric field is applied between the electrodes of a rigidly clamped film, a sinusoidal stress is generated in the sample. From the observation of such inverse piezoelectric effect, the piezoelectric stress-constant $e$ can be determined. ${ }^{16}$ Figure 8 illustrates the anisotropy of $e$ for the $\mathrm{PVF}_{2}$ film 105 day after polarization. The variation of $e$ with $\theta$ was represented by a form similar to eq 2 , i.e.,

$$
e=e_{31} \cos ^{2} \theta+e_{32} \sin ^{2} \theta
$$

The piezoelectric stress-constant $e$ should also be considered as a complex quantity and is expressed by $e=e^{\prime}-i e^{\prime \prime}$. The separate determination of $e^{\prime}$ and $e^{\prime \prime}$ has not yet been carried out. The relation between $d$ and $e$ is given by

$$
e=C^{E} d
$$

where $C^{E}$ is an elastic constant at $E=0$. Young's moduli of the sample were experimentally determined as $C_{11}^{E}=9.4 \times 10^{9} \mathrm{dyn} / \mathrm{cm}^{2}$ and $C_{22}^{E}=7.5 \times 10^{9} \mathrm{dyn} / \mathrm{cm}^{2}$. The results agreed with those calculated by eq 8 from the observed values of $e$ (Figure 8 ) and $d$ (Figure 3 ).

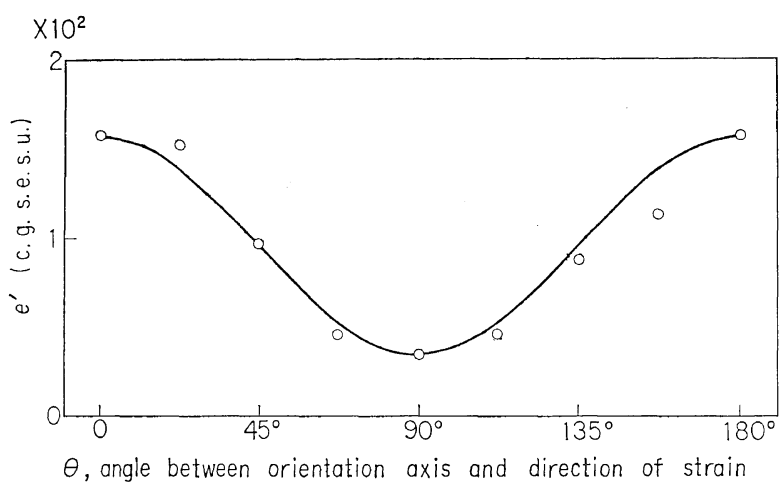

Figure 8. Dependence of the real piezoelectric stress-constant $e^{\prime}$ on the angle between the direction of strain and the axis of elongation. 
The anisotropy of $d$ and $e$ can be understood from the symmetry of the elongated and polarized film, i.e., $C_{2 v}$ or $\mathrm{mm} \mathrm{2.} \mathrm{The} \mathrm{matrix} \mathrm{of}$ the piezoelectric strain-constant for this class of symmetry is given by, ${ }^{17}$

$\begin{array}{cccccc}0 & 0 & 0 & 0 & d_{15} & 0 \\ 0 & 0 & 0 & d_{24} & 0 & 0 \\ d_{31} & d_{32} & d_{33} & 0 & 0 & 0\end{array}$

The relation between $d$ and $\theta$, the angle between the direction of stress and the $x$-axis, is derived from this matrix as follows

$$
d=d_{31} \cos ^{2} \theta+d_{32} \sin ^{2} \theta+d_{36} \sin \theta \cos \theta
$$

The fact that the experimental results were well represented by eq 2 indicates $d_{36}=0$. Sheartype piezoelectricity, as observed in the elongated polypeptides, does not exist.

The piezoelectric constant, $d_{33}$, has been qualitatively confirmed by the observation of the electric voltage produced under the applied pressure on the surface of the film. At present no observation is possible to examine the constants $d_{15}$ and $d_{24}$, because the sample is in the shape of thin film.

The value of $d_{31}^{\prime}$ at room temperature is around $10^{-7} \mathrm{cgs}$ esu, depending on the conditions of elongation and poling processes. According to Kawai, ${ }^{6}$ the piezoelectric strain-constant $d_{31}$ is represented by

$$
d_{31}=2 \gamma^{T}{ }_{31} E_{S}
$$

where, $\gamma_{31}^{T}$ is the electrostriction constant at constant stress and $E_{S}$ is the spontaneous electric field present in the sample. The origin of $E_{S}$ will be either the oriented dipoles or the antisymmetrically distributed space charges produced during the electret-forming procedures. It has been confirmed ${ }^{6,16}$ that the signs of both $\gamma_{31}^{T}$ and $d_{31}$ are positive. Accordingly the sign of $E_{S}$ becomes positive.

In a previous report, ${ }^{9}$ it was stated that the constant $d_{31}$ decreased at about $80^{\circ} \mathrm{C}$, where the poling process was performed. However, Figure 5 indicates that $d_{31}$ does not vanish at $80^{\circ} \mathrm{C}$ and that the film remains piezoelectric above its poling temperature. It seems that as long as the homocharges are retained the piezoelectric effect does not disappear.

If the piezoelectric effect is produced by the combination of electrostriction and the spontaneous polarization due to the homocharge, the temperature variation of $d_{31}$ is mainly determined by the temperature dependence of the electrostriction constant $\gamma_{31}^{T}$. Figure 5 should then indicate the temperature dependence of $\gamma_{31}^{T}$. It is interesting to note that the phase angle between the polarization and the stress is always in lagging phase and therefore no negative values of $d_{31}^{\prime \prime}$ and $d_{32}^{\prime \prime}$ are observed. This is also quite different from the results observed in the previously known piezoelectric polymers such as polypeptides. ${ }^{3}$

The phase angle in the piezoelectric constant $d_{31}$ is larger than that in the elastic constant $E_{1}$; for example, at room temperature, $\tan \delta$ $\left(d_{31}\right)=0.08$ and $\tan \delta\left(E_{1}\right)=0.035$. This suggests that the phase lag in piezoelectricity is not only produced from the viscoelastic phase lag.

Electrostriction is expected to occur both in the crystalline and amorphous regions of the polymer. No information is available at present on the influence of crystallinity on the electrostriction of the polymer.

It has been reported that the strong stretch of the film is favorable for obtaining high piezoelectric effect. The same poling procedure as to the elongated film was given to the original $\mathrm{PVF}_{2}$ film. After the procedure, the film acquired piezoelectric property and its anisotropy is shown in Figure 9. Here the angle

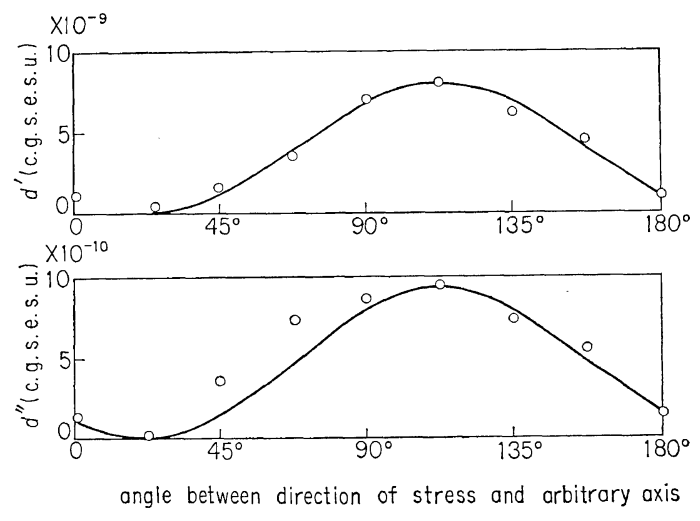

Figure 9. Dependence of the piezoelectric strainconstants $d^{\prime}$ and $d^{\prime \prime}$ on the direction of stress in the plane of the cast film. The direction that the angle is $112^{\circ}$ should be the direction of naturally existing orientation. 


\section{E. Fukada and T. Sakurai}

$\theta$ was measured from an arbitrarily determined direction on the surface of the film. Similar anisotropy as in Figure 3 was observed, though the largest value of $d^{\prime}$ was only $8 \times 10^{-9} \mathrm{cgs}$ esu. This anisotropy may be due to the original orientation of the $\mathrm{PVF}_{2}$ molecules which were produced during the formation of the film. Since the magnitude of the electrostriction constant will change little due to elongation, ${ }^{6}$ the decrease of $d^{\prime}$ should be related to the decrease of the spontaneous electric field, or to the density of the space charge. It is likely that the highly elongated planar zigzag conformation of the $\mathrm{PVF}_{2}$ molecule, particularly the $\beta$-form crystals, ${ }^{18,19}$ is strongly favorable to trapping the homocharge. Since the $\mathrm{CF}_{2}$ dipoles are normally alligned to the molecular chain axis in the $\beta$-form crystallites, the preferred orientation of the dipoles would lie normal to the surface of the film. Such a local electric field of the dipoles might be responsible for traping a large amount of homocharge. A detailed investigation of the electrostriction and its temperature dependence will be published shortly.

Note Added in Proof. According to experimental findings after submitting the present paper, the authors reached an interpretation for the spontaneous polarization different from that given in the paper. Firstly, the piezoelectric effect can be detected even when the surface charge of the electret film is no longer observed. Secondly, the magnitude of spontaneous electric field $E_{\mathrm{s}}$, calculated by eq 10 from experimental values of $d_{31}$ and $\gamma_{31}^{T}$, increases during the poling process far beyond the electric field applied to the film. ${ }^{16}$ These observations may be properly explained if we rssume that the spontaneous polarization, associated with a spontaneous strain, has been produced by the orientation of dipoles. Denoting the spontaneous strain $S_{\mathrm{s}}=\gamma_{31}^{T} E_{\mathrm{s}}{ }^{2}$, eq 10 is transformed to $d_{31}=2 \sqrt{\gamma_{31}^{T} S_{\mathrm{s}}}$. Piezoelectricity can be produced by the variation with stress of $S_{\mathrm{s}}$ which is due to oriented dipoles in the absence of space charges. During the poling process $S_{\mathrm{s}}$ gradually increases due to the progress of the orientation of dipoles, which results in the increase of apparent spontaneous field $E_{\mathrm{s}}=\sqrt{S_{\mathrm{s}} / \gamma_{31}^{T}}$.

Acknowledgement. The authors thank Prof. H. Kawai of Yokohama City University for helpful discussions and Dr. M. Asahina of Kureha Chemical Industry Co., Ltd. for providing us with the $\mathrm{PVF}_{2}$ specimens.

\section{REFERENCES}

1. E. Fukada, M. Date, and K. Hara, Japan. $J$. Appl. Phys., 8, 151 (1969).

2. E.Fukada and I.Yasuda, Japan. J. Appl. Phys., 3, 117 (1964).

3. M. Date, S. Takashita, and E. Fukada, $J$. Polym. Sci., Part A-2, 8, 61 (1970).

4. T. Furukawa and E. Fukada, Nature, 221, 1235 (1969).

5. H. Kawai, Japan. J. Appl. Phys., 8, 975 (1969).

6. H. Kawai Ohyobutsuri, 38, 1133 (1969); 39, 413 (1970); 39, 869 (1970).

7. K. Nakamura and Y. Wada, Rept. Progr. Polym. Phys. Japan, 13, 383, 387 (1970).

8. S. Edelman, L. R. Grisham, S. C. Roth, and J. Cohen, J. Acous. Soc. Amer., 48, 1040 (1970).

9. E. Fukada, S. Takashita, Japan. J. Appl. Phys. 8, 960 (1969).

10. B. Gross, "Charge Storage in Solid Dielectrics," Elsever, Amsterdam, 1964.

11. L. M. Baxt and M. M. Perlman, "Eletrets and Related Electrostatic Charge Storage Phenomena," Symp. Ser. Electrochemical Society Ed., New York, N.Y., 1968.

12. T. Takamatsu and E. Fukada, Riken Hokoku (Rept. Inst. Phys. Chem. Res.), 45, 1 (1969). (in Japanese)

13. H. Sasabe, S. Saito, M. Asahina, and H. Kakutani, J. Polym. Sci., Part A-2, 7, 1405 (1969).

14. H. Kakutani, J. Polym, Sci., Part A-2, 8, 1177 (1970).

15. M. Date, and E. Fukada, Rept. Progr. Polym. Phys. Japan, 7, 183 (1964).

16. M. Oshiki and E. Fukada, under preparation.

17. W. G. Cady, "Piezoelectricity," Dover, Publishers, Inc., New York, N. Y., 1964, p 191.

18. J. B. Lando, H. G. Olf, and A. Peterlin, J. Polym. Sci., Part A-1, 4, 941 (1966).

19. J. B. Lando, W. W. Doll, J. Macromol. Sci.Phys., B2 (2), 205 (1968). 